Geometry $\mathcal{G}^{\mathcal{T}}$ Topology

Volume 6 (2002) 495-521

Published: 22 November 2002

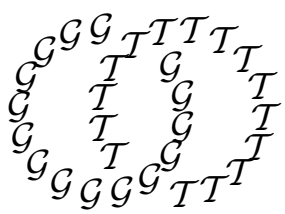

\title{
Lengths of simple loops on surfaces with hyperbolic metrics
}

\author{
FENG LUO \\ RichARD STONG \\ Department of Mathematics, Rutgers University \\ New Brunswick, NJ 08854, USA \\ and \\ Department of Mathematics, Rice University \\ Houston, TX 77005, USA \\ Email: fluo@math.rutgers.edu and stong@math.rice.edu
}

\begin{abstract}
Given a compact orientable surface of negative Euler characteristic, there exists a natural pairing between the Teichmüller space of the surface and the set of homotopy classes of simple loops and arcs. The length pairing sends a hyperbolic metric and a homotopy class of a simple loop or arc to the length of geodesic in its homotopy class. We study this pairing function using the Fenchel-Nielsen coordinates on Teichmüller space and the Dehn-Thurston coordinates on the space of homotopy classes of curve systems. Our main result establishes Lipschitz type estimates for the length pairing expressed in terms of these coordinates. As a consequence, we reestablish a result of ThurstonBonahon that the length pairing extends to a continuous map from the product of the Teichmüller space and the space of measured laminations.
\end{abstract}

AMS Classification numbers Primary: 30F60

Secondary: 57M50, 57N16

Keywords: Surface, simple loop, hyperbolic metric, Teichmüller space

Proposed: David Gabai

Seconded: Jean-Pierre Otal, Joan Birman
Received: 20 April 2002

Revised: 19 November 2002

(C) Geometry $\& \mathcal{T}$ Topology Publications 


\section{Introduction}

1.1 Given a compact orientable surface of negative Euler characteristic, there exists a natural length pairing between the Teichmüller space of the surface and the set of homotopy classes of simple loops and arcs. The length pairing sends a hyperbolic metric and a homotopy class of a simple loop or arc to the length of the geodesic in its homotopy class. In this paper, we study this pairing function using the Fenchel-Nielsen coordinates on Teichmüller space and the Dehn-Thurston coordinates on the space of homotopy classes of curve systems. Our main result, theorem 1.1, establishes Lipschitz type estimates for the length pairing expressed in terms of these coordinates. As a consequence, we give a new proof of a result of Thurston-Bonahon ([13], see [2, proposition 4.5] for a proof) that the length pairing extends to a continuous map from the product of the Teichmüller space and the space of measured laminations to the real numbers so that the extension is homogeneous in the second coordinate.

1.2 Let $F$ be a compact connected orientable surface with possibly non-empty boundary and negative Euler characteristic. By a hyperbolic metric on the surface $F$ we mean a Riemannian metric of curvature -1 on the surface $F$ so that its boundary components are geodesics. The Teichmüller space $T(F)$ is the space of all isotopy classes of hyperbolic metrics on the surface. Recall that two hyperbolic metrics are isotopic if there is an isometry between the two metrics which is isotopic to the identity. Following M. Dehn [5], a curve system in the surface $F$ is a compact proper 1-dimensional submanifold so that each of its circle components is not null homotopic and not homotopic into the boundary $\partial F$ of $F$ and each of its arc component is not homotopic into $\partial F$ relative to its endpoints. We denote the set of all homotopy classes (or equivalently isotopy classes) of curve systems on $F$ by $C S(F)$ and call it the space of curve systems. By a basic fact from hyperbolic geometry, for any hyperbolic metric $d$ on $F$ and any homotopically non-trivial simple loop or $\operatorname{arc} s$ in $F$, there is a unique shortest $d$-geodesic $s^{*}$ homotopic (and isotopic) to $s$. One defines the length of the homotopy class $[s]$, denoted by $l_{d}([s])$ (or $l_{[d]}([s]$ ) since it depends only on the class $[d] \in T(F)$ ), to be the $d$-length of the geodesic $s^{*}$. This length pairing extends naturally to a map $T(F) \times C S(F) \rightarrow \mathbb{R}$, still denoted by $l_{d}([s])$.

Our goal is to understand this length pairing using parametrizations of $T(F)$ and $C S(F)$. To this end, let us recall the Fenchel-Nielsen coordinates on Teichmüller space and Dehn-Thurston coordinates on the space of curve systems. The definition of these two coordinates depends on the choice of a hexagonal decomposition on the surface (see section 2.2). Fix such a decomposition on a surface of genus $g$ with $r$ boundary components, we obtain a parametrization 
(the Fenchel-Nielsen coordinates) of the Teichmüller space $F N: T(F) \rightarrow R$ where $R=\left(\mathbb{R}_{>0} \times \mathbb{R}\right)^{3 g-r+3} \times \mathbb{R}_{>0}^{r}$ and a parametrization (the Dehn-Thurston coordinates) DT:CS(F) $\rightarrow Z$ where $Z=((\mathbb{Z} \times \mathbb{Z}) / \pm)^{3 g-r+3} \times \mathbb{Z}_{>0}^{r}$. (See section 2 and section 3 for details). Here $\mathbb{R}_{>0}$ and $\mathbb{Z}_{>0}$ denote the sets of positive real numbers and positive integers respectively. Note that $F N$ is a homeomorphism and $D T$ is an (homogeneous) injective map.

We introduce a metric on the space $Z$ as follows. The metric on $(\mathbb{Z} \times \mathbb{Z}) / \pm$ is defined to be $\left|\left(x_{1}, y_{1}\right)-\left(x_{2}, y_{2}\right)\right|=\min \left\{\left|x_{1}+x_{2}\right|+\left|y_{1}+y_{2}\right|,\left|x_{1}-x_{2}\right|+\left|y_{1}-y_{2}\right|\right\}$. The metric on $\mathbb{Z}_{>0}$ is the standard metric and the metric on $Z$ is the product metric. The length $|x|$ of $x=\left(\left[x_{1}, t_{1}\right], \ldots,\left[x_{N}, t_{N}\right], x_{N+1}, \ldots, x_{N+r}\right) \in Z$ is $\sum_{i=1}^{N+r}\left|x_{i}\right|+\sum_{j=1}^{N}\left|t_{j}\right|$ where $N=3 g+r-3$.

For $x=\left(x_{1}, t_{1}, \ldots, x_{N}, t_{N}, x_{N+1}, \ldots, x_{N+r}\right)$ and

$$
\begin{aligned}
y & =\left(y_{1}, s_{1}, \ldots, y_{N}, s_{N}, y_{N+1}, \ldots, y_{N+r}\right) \text { in } R, \text { let } \\
D(x, y)= & \sum_{i=1}^{N} \min \left\{x_{i}, y_{i}\right\}\left|t_{i}-s_{i}\right|+ \\
& \quad\left(5 \max _{i}\left\{\left|t_{i}\right|,\left|s_{i}\right|\right\}+7\right) \sum_{i=1}^{N+r}\left|\log \sinh \left(x_{i} / 2\right)-\log \sinh \left(y_{i} / 2\right)\right| .
\end{aligned}
$$

Note that this $D: R \times R \rightarrow \mathbb{R}$ is continuous and satisfies $D(x, y)>0$ if $x \neq y$, but it is not a metric on $R$. Define

$$
|x|=\sum_{i=1}^{N}\left(x_{i}+1 / x_{i}+x_{i}\left|t_{i}\right|\right)+\sum_{j=N+1}^{N+r}\left(x_{j}+1 / x_{j}\right)+(N+r) \log 2 .
$$

Here $x_{i}$ is the length of the $i$-th decomposing loop in the metric and $x_{i} t_{i}$ is the twisting length. The number $2 \pi t_{i}$ measures the angle of twisting at the $i$-th decomposing loop.

Our main theorem is the following.

Theorem 1.1 Suppose $F$ is a compact orientable surface with possibly nonempty boundary components and the surface $F$ has a fixed hexagonal decomposition. Let $F N: T(F) \rightarrow\left(\mathbb{R}_{>0} \times \mathbb{R}\right)^{3 g-r+2} \times \mathbb{R}_{>0}^{r}$ and DT:CS(F) $\rightarrow$ $((\mathbb{Z} \times \mathbb{Z}) / \pm)^{3 g-r+2} \times \mathbb{Z}_{>0}^{r}$ be the Fenchel-Nielsen coordinate and the DehnThurston coordinate associated to the hexagonal decomposition. Then for any $[a],[b]$ in $C S(F)$ and any two hyperbolic metrics $\left[d_{1}\right],\left[d_{2}\right]$ in $T(F)$, the following inequalities hold.

$$
\left|l_{d_{1}}([a])-l_{d_{1}}([b])\right| \leq 3\left|F N\left(d_{1}\right)\right||D T([a])-D T([b])|,
$$

and

$$
\left|l_{d_{1}}([a])-l_{d_{2}}([a])\right| \leq 4 D\left(F N\left(d_{1}\right), F N\left(d_{2}\right)\right)|D T([a])| .
$$


As a consequence, we give a new proof of the following result of ThurstonBonahon (see [2] for the first published proof).

Corollary 1.2 ([13], [2]) The hyperbolic length function extends to a continuous map from $T(F) \times M L(F) \rightarrow \mathbb{R}$ where $M L(F)$ is the space of measured laminations on the surface $F$. Furthermore, the extension also satisfies the inequalities (1.1) and (1.2).

1.3 One of the main ingredients used in the proof is the following elementary geometric fact about right-angled hyperbolic hexagons (see theorem 5.2 in section 5). Let $H_{x, a, b}$ be a right-angled hyperbolic hexagon whose side lengths are (reading from counterclockwise): $a, z, x, y, b, w$. Let $S_{\lambda, \mu}$ be the length of a geodesic segment in $H_{x, a, b}$ joining any two sides of the hexagon so that the endpoints of the segment cut the sides into two intervals of lengths $\lambda t,(1-\lambda) t$ and $\mu s,(1-\mu) s$. Then if we fix $a, b, \lambda, \mu$ and let $x$ vary, the length $S_{\lambda, \mu}$ satisfies

$$
\left|\frac{d S_{\lambda, \mu}}{d x}\right| \leq 4 \operatorname{coth} x .
$$

In particular, this implies that,

$$
\left|S_{\lambda, \mu}(x)-S_{\lambda, \mu}\left(x^{\prime}\right)\right| \leq 4\left|\log \sinh (x)-\log \sinh \left(x^{\prime}\right)\right| .
$$

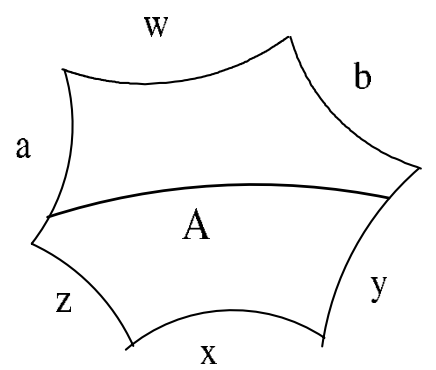

Figure 1.1

1.4 The paper is organized as follows. In section 2, we recall some of the known facts about the curve systems and the results obtained in [10]. In particular, we will recall the notion of the hexagonal decompositions of the surface and the Dehn-Thurston coordinates on the space of curve systems. In section 3, we will recall the Fenchel-Nielsen coordinates of hyperbolic metrics. The main theorem 1.1 will be proved in section 4 . In section 5, we establish two simple facts on hyperbolic right angled hexagon used in the proof.

The work is supported in part by the NSF. 


\section{Dehn-Thurston coordinates of curve systems}

We will recall the Dehn-Thurston coordinates on $C S(F)$ in this section. The basic ingredient to set up the coordinate is the colored hexagonal decomposition of a surface which is defined in subsection 2.1 below. Unless mentioned otherwise, we will assume in this section that the surface $F$ is oriented with negative Euler characteristic.

\subsection{Notation and conventions}

We shall use the following notations and conventions. Let $F=F_{g, r}$ be the orientable compact surface of genus $g$ with $r \geq 0$ boundary components. The interior of a surface $F$ will be denoted by $\operatorname{int}(F)$. All subsurfaces in an oriented surface have the induced orientation. We will always draw oriented surface so that its orientation is the right-hand orientation on the front face of the surface that we see.

A curve system on $F$ is a proper 1-dimensional submanifold $s$ in $F$ so that no circle component of $s$ is null homotopic or homotopic into the boundary of the surface $F$ and no arc component of $s$ is null homotopic relative to the boundary. If $s$ is a proper submanifold of a surface, we use $N(s)$ to denote a small tubular neighborhood of $s$. The isotopy class of a submanifold $s$ is denoted by $[s]$. If $a$ and $b$ are isotopic submanifolds we will write $a \cong b$. If $a, b$ are two proper 1-dimensional submanifolds, we will use $I(a, b), I([a], b)$ or $I(a,[b])$ to denote the geometric intersection number $I([a],[b])=\min \left\{\left|a^{\prime} \cap b^{\prime}\right|: a \cong a^{\prime}, b \cong b^{\prime}\right\}$. Here $|X|$ denoted the cardinal of a set $X$. When a curve system $a$ is written as a union $a_{1} \cup \ldots \cup a_{n}$, it is understood that each $a_{i}$ is a union of components of $a$. Let $2 \mathbb{Z}$ be the set of even integers. All hyperbolic metrics on compact surfaces are assumed to have geodesic boundary. Also if $d$ is a hyperbolic metric and $a$ is a curve system, we use $l_{d}(a)$ to denote the length of $a$ in the metric $d$. The length of the isotopy class $[a]$ is defined to be $\inf \left\{l_{d}\left(a^{\prime}\right) \mid a^{\prime} \cong a\right\}$ and is denoted by $l_{d}([a])$.

Fix an orientation on the surface $F$. Let us recall the concept of multiplication of two curve systems in $C S(F)$ (see [3], [11] and [9], the notation was first introduced in [11], [3] as the earthquakes in the space of measured laminations). Given $\alpha$ and $\beta$ in $C S(F)$, take $a \in \alpha$ and $b \in \beta$ so that $|a \cap b|=I(\alpha, \beta)$. If $\alpha$ and $\beta$ are disjoint, we define $\alpha \beta$ to be $[a \cup b]$. If $I(\alpha, \beta)>0$, then $\alpha \beta$ is defined to be the isotopy class of the 1 -dimensional submanifold $a b$ obtained by resolving all intersection points in $a \cap b$ from $a$ to $b$. Here by the resolution from $a$ to $b$ we mean the following surgery. At each point $p \in a \cup b$, fix any orientation on $a$. Then use the orientation of the surface to determine an orientation of 
$b$ at $p$. Finally resolve the singularity at $p$ according to the orientations on $a$ and $b$. One checks easily that this is independent of the choice of orientation on $a$. See figure 2.1. If $a$ is a curve system and $k$ is a positive integer, then the collection of $k$ parallel copies of $a$ is denoted by $a^{k}$. We use $[a]^{k}$ to denote $\left[a^{k}\right]$. If $k$ is a negative integer, we denote $[a]^{k}[b]$ by $[b][a]^{-k}$ and $[b][a]^{k}$ by $[a]^{-k}[b]$.
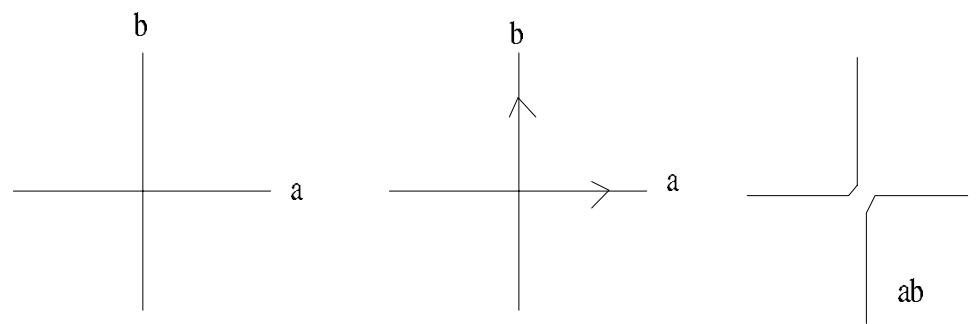

Figure 2.1

The following useful property follows from the definition.

Lemma 2.1 (Triangle inequality) Suppose $a$ is a curve system without arc components and $b$ is a curve system. Fix a hyperbolic metric $d$ on the surface $F$. Then the hyperbolic lengths satisfy

and

$$
\left|l_{d}([a b])-l_{d}([b])\right| \leq l_{d}([a]),
$$

$$
\left|l_{d}([b a])-l_{d}([b])\right| \leq l_{d}([a]) .
$$

Indeed, by the definition of resolutions and taking all components of $a$ and $b$ to be geodesics, one sees that $l_{d}([a b]) \leq l_{d}([a])+l_{d}([b])$ (this inequality also holds for curve systems $a$ with arc components). To see the inequality $l_{d}([b]) \leq$ $l_{d}([a b])+l_{d}([a])$, we use the cancelation property of the multiplication ([10] theorem 2.4(4)) that $(a b) a \cong b \cup c^{2}$ where $c$ consists of those components of $a$ which are disjoint from $b$. Thus $l_{d}([b]) \leq l_{d}\left(\left[b \cup c^{2}\right]\right)=l_{d}([(a b) a]) \leq$ $l_{d}([a b])+l_{d}([a])$. This proves the lemma.

A curve system $s$ on $F$ is called a 3-holed sphere decomposition if (1) each component of $s$ is a circle and (2) all components of $F-s$ are 3 -holed spheres. This implies that $s$ contains $3 g+r-3$ many components when $F=F_{g, r}$.

By a hexagonal decomposition of the 3 -holed sphere $F_{0,3}$, we mean a curve system $b$ on $F_{0,3}$ so that $b$ contains exactly three arc components joining different boundary components in $F_{0,3}$. See figure 2.2(a). We call each component of $F_{0,3}-b$ a hexagon. A colored hexagonal decomposition of an orientable compact 
surface $F$ is a triple $(p, b, c o l)$ where $p, b$ are curve systems and col is a coloring so that (1) $p$ is a 3 -holed sphere decomposition, (2) for each component $F^{\prime}$ of $F-p$, the intersection $b \cap F^{\prime}$ is a hexagonal decomposition of the 3-holed sphere, (3) one can color the components of $F-p \cup b$ into red and white so that there is exactly one red hexagon in each component of $F-p$ and the red hexagons join only red hexagons crossing $p$. The triple $(p, b, c o l)$ is also called a marking on the surface $F$.

2.2 The classification of the curve systems on the 3 -holed sphere $F_{0,3}$ is well known. Suppose the boundary components of the 3 -holed sphere $F_{0,3}$ are $\partial_{1}, \partial_{2}, \partial_{3}$. Then each $[a] \in C S\left(F_{0,3}\right)$ is determined uniquely by $\operatorname{DT}([a])=$ $\left(x_{1}, x_{2}, x_{3}\right)$ where $x_{i}=I\left(a, \partial_{i}\right)$. Furthermore the map DT:CS(F,3) $\rightarrow$ $\left\{\left(x_{1}, x_{2}, x_{3}\right) \in \mathbb{Z}_{\geq 0}^{3} \mid x_{1}+x_{2}+x_{3} \in 2 \mathbb{Z}\right\}$ is a bijection. These are the DehnThurston coordinates for the 3 -holed sphere. The curve systems with coordinates $\left(x_{1}, x_{2}, x_{3}\right)$ are shown in figure $2.2(\mathrm{~b})$.

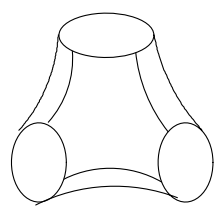

(a)
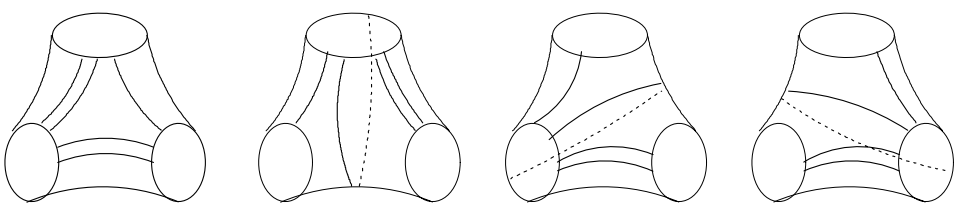

(b)
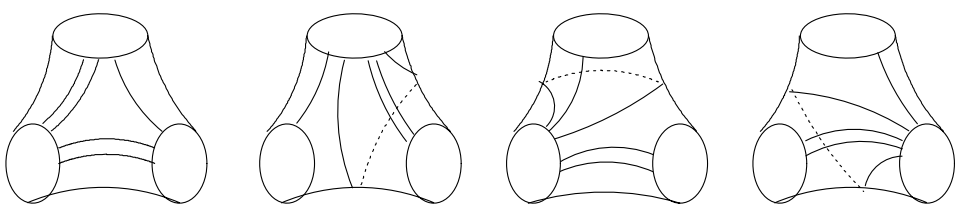

(c)

Figure 2.2

If we fix a colored hexagonal decomposition $b=b_{1} \cup b_{2} \cup b_{3}$ of the oriented surface $F_{0,3}$, then each $[a] \in C S\left(F_{0,3}\right)$ has a standard representative with respect to 
the hexagonal decomposition. It is defined as follows. We assume that $b_{i}$ is disjoint from $\partial_{i}$. Take a curve system $a$ in $F_{0,3}$. Its standard representative is a curve system $a^{\prime} \cong a$ so that each component of $a^{\prime}$ is standard. Here an arc $s$ is standard if either it lies entirely in the red-hexagon or if $\partial s \subset \partial_{i}$, then $\partial s$ is in the red-hexagon and $\left|s \cap\left(b_{1} \cup b_{2} \cup b_{3}\right)\right|=2=\left|s \cap\left(b_{i} \cup b_{j}\right)\right|$ so that the cyclic order of the sets $\left(s \cap \partial_{i}, s \cap b_{i}, s \cap b_{j}\right)$ in the boundary of the red-hexagon coincides with the induced orientation from the red-hexagon. For instance the standard representatives of the curve systems with coordinates $\left(x_{1}, x_{2}, x_{3}\right)$ are shown in figure 2.2(c) where the red-hexagon is the front hexagon in figure $2.2(\mathrm{a})$.

Fix a marking $\left(p_{1} \cup \ldots \cup p_{3 g+r-3}, b, c o l\right)$ on an oriented surface $F=F_{g, r}$. The Dehn-Thurston coordinates of $[a]$ in $C S(F)$ is a vector in $\left(\mathbb{Z}^{2} / \pm\right)^{3 g+r-3} \times \mathbb{Z}_{\geq 0}^{r}$ defined as follows. Express the class $[a]$ as

$$
[a]=\left[p_{1}^{t_{1}} \ldots p_{3 g+r-3}^{t_{3 g+r-3}}\right]\left[a_{z t}\right]
$$

where $t_{i} \in \mathbb{Z}$ so that if $I\left(a, p_{i}\right)=0$ then $t_{i} \geq 0$ and $a_{z t}$ is a curve system so that its restriction to each 3 -holed sphere component of $F-p_{1} \cup \ldots \cup p_{3 g+r-3}$ is a standard curve system with respect to the red hexagon. Then the DehnThurston coordinate of $[a]$ is

$$
D T([a])=\left(\left[x_{1}, t_{1}\right], \ldots,\left[x_{3 g+r-3}, t_{3 g+r-3}\right], x_{3 g+r-2}, \ldots, x_{3 g+2 r-3}\right)
$$

where $x_{i}=I\left(a, p_{i}\right)$ and $p_{3 g+r-3+j}=\partial_{j} F$. Note that $I\left(a, p_{i}\right)=I\left(a_{z t}, p_{i}\right)$ and the twisting coordinates $t_{i}\left(a_{z t}\right)$ of $a_{z t}$ are zero. We sometimes use $x_{i}(a)$ and $t_{j}(a)$ to denote the coordinates $x_{i}$ and $t_{j}$ of the curve systems $a$. It is shown in [10] (proposition 2.5) that this is well defined. For $[s] \in C S(F)$ and $k \mathbb{Z}_{>0}$, let $[s]^{k}=\left[s^{k}\right]$ be the isotopy class of $k$-parallel copies of $s$.

Proposition 2.2 The Dehn-Thurston coordinate is a bijection

$$
\begin{aligned}
& \text { DT: } C S(F) \rightarrow \\
& \quad\left\{\left(\left[x_{1}, t_{1}\right], \ldots,\left[x_{3 g+r-3}, t_{3 g+r-3}\right], x_{3 g+r-2}, \ldots, x_{3 g+2 r-3}\right) \in\left(\mathbb{Z}^{2} / \pm\right)^{2 g+r-3}\right. \\
& \left.\times\left(\mathbb{Z}_{\geq 0}\right)^{r} \mid \text { if } p_{i}, p_{j} \text { and } p_{k} \text { bound a } 3 \text {-holed sphere, then } x_{i}+x_{j}+x_{k} \in 2 \mathbb{Z}\right\} .
\end{aligned}
$$

Furthermore, $D T\left([a]^{k}\right)=k D T([a])$ for $k \in \mathbb{Z}_{\geq 0}$.

\subsection{The main idea of the proof of theorem 1}

We sketch the proof of the inequality (1.1) in the main theorem 1.1 in this subsection. First of all, by homogeneity $l_{d}\left(\left[a^{2}\right]\right)=2 l_{d}([a])$ and $D T\left(a^{2}\right)=$ $2 D T(a)$, hence it suffices to prove (1.1) for classes $[a],[b]$ so that $D T(a)=u$ and $D T(b)=v$ are even vectors, ie, all $x_{i}$ and $t_{j}$ coordinates of them are even 
integers. Now given any two even vectors $u$ and $v$ in $Z$ with distance $|u-v|=$ $2 n$ there exists a sequence of $n+1$ even vectors $u_{0}=u, u_{1}, \ldots, u_{n}=v$ so that $\left|u_{i}-u_{i+1}\right|=2$. On the other hand, by proposition 2.2 , each even vector $u_{i}$ is the image $D T\left(a_{i}\right)$ for some $\left[a_{i}\right] \in C S(F)$. Thus by interpolation, it suffices to prove inequality (1.1) for classes $[a]$ and $[b]$ so that $D T(a)$ and $D T(b)$ are even vectors of distance two apart. This means that the Dehn-Thurston coordinates of $[a]$ and $[b]$ are the same except at one $x_{i}-$ or $t_{j}$-coordinate where they differ by 2 . If one of their twisting coordinates differs by 2 , say $t_{i}(a)=t_{i}(b)+2$, then $[a]=\left[p_{i}^{2} b\right]$ by definition. Thus, by the triangle inequality (Lemma 2.1), we have $\left|l_{d}([a])-l_{d}([b])\right| \leq l_{d}\left(\left[p_{i}^{2}\right]\right)=2 l_{d}\left(\left[p_{i}\right]\right) \leq|F N(d)||D T(a)-D T(b)|$. If their intersection number coordinates differ by two, say $x_{i}(a)=x_{i}(b)+2$, for some $i$ with $1 \leq i \leq 3 g+r-3$, then we prove in [10] (proposition 4.3) that $[a]=\delta_{1} \ldots \delta_{s}[b] \delta_{s+1} \ldots \delta_{t}$ where $t \leq 5$ and the $\delta_{i}$ 's are quite simple. In fact, we show that these simple loops $\delta_{i}$ 's satisfy

$$
\sum_{i=1}^{t} l_{d}\left(\delta_{i}\right) \leq 6|F N(d)| .
$$

Thus by the triangle inequality (lemma 2.1), $\left|l_{d}([a])-l_{d}([b])\right| \leq \sum_{i=1}^{t} l_{d}\left(\delta_{i}\right) \leq$ $6|F N(d)|=3|F N(d)||D T(a)-D T(b)|$. If their intersection number coordinates differ by two $x_{i}(a)=x_{i}(b)+2$ for some $i$ with $i \geq 3 g+r-2$, then doubling the surface across its boundary reduces to the previous case.

This shows that the main issue is to understand the effect of changing some intersection coordinate $x_{i}$ by 2 . This will be addressed in the following subsections.

2.4 We will recall the results obtained in [10] concerning the change of $x_{i}$ coordinates by 2 . Suppose $\left(p_{1} \cup \ldots \cup p_{3 g+r-3}, b, c o l\right)$ is a marking on an oriented surface $F$, and $D T$ is the associated Dehn-Thurston coordinate. Let $[a]$ and [b] be two isotopy classes of curve systems so that their twisting coordinates $t_{j}(a)$ and $t_{j}(b)$ are the same and their intersection coordinates agree except for the $i$-th which satisfies $x_{i}(a)=x_{i}(b)+2$. We will find a surgery procedure converting $a$ to $b$. There are three cases to be discussed. In the first case, the corresponding decomposing simple loop $p_{i}$ is adjacent to only one 3 -holed sphere component of $F-p$ and $p_{i}$ is not in $\partial F$. In the second case, the simple loop $p_{i}$ is adjacent to two different components of $F-p$. In the last case, $p_{i}$ is a boundary component of the surface $F$.

The following two results were obtained in [10] (propositions 4.2 and 4.3). 


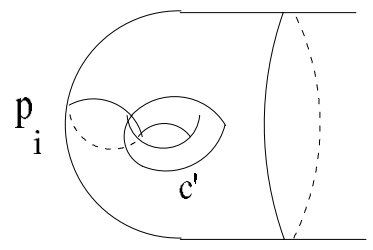

$\mathrm{p}_{\mathrm{j}}$

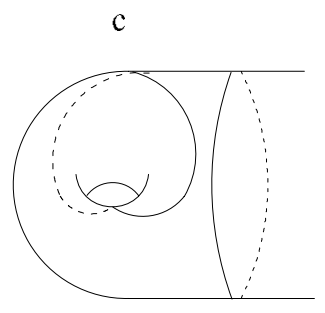

$\mathrm{p}_{\mathrm{j}}$ $\mathrm{c}$

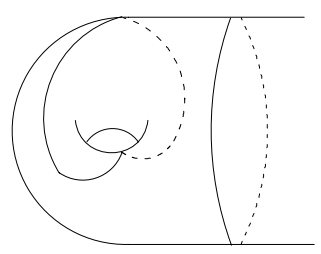

$\mathrm{p} j_{\mathrm{j}}$

Figure 2.3: Here $c^{\prime}$ is the simple loop with zero twisting coordinate.

The loop $c$ is obtained from $c^{\prime}$ by a Dehn twist along $p_{i}$.

Proposition 2.3 ([10], proposition 4.2) In the first case that $p_{i}$ is adjacent to only one 3 -holed sphere, suppose $p_{j}$ is the simple loop bounding the 1-holed torus which contains $p_{i}$. Then

$$
a \cong p_{j}^{e_{1}} c^{e_{2}} b
$$

where $e_{1}, e_{2} \in\{0, \pm 1, \pm 2\}$ and $c$ is one of the two simple loops with DehnThurston coordinates $([0,0], \ldots,[0,0],[1, \pm 1],[0,0], \ldots,[0,0], 0, \ldots, 0)$ (the nonzero coordinates are $x_{i}$ and $\left.t_{i}\right)$. See figure 2.3 .

Proposition 2.4 ([10], proposition 4.3) In the second case that $p_{i}$ is adjacent to two 3 -holed spheres, suppose $p_{i_{1}}, \ldots, p_{i_{4}}$ are the simple loops bounding the 4-holed sphere containing $p_{i}$ and $p_{i}, p_{i_{1}}, p_{i_{2}}$ bound a 3 -holed sphere. Then

$$
a \cong p_{i_{1}}^{s_{1}} \ldots p_{i_{4}}^{s_{4}} c^{e} b
$$

where $e \in\{ \pm 1\},\left|s_{1}\right|+\left|s_{2}\right| \leq 2,\left|s_{3}\right|+\left|s_{4}\right| \leq 2$ and $c$ is a simple loop in the 4holed sphere whose Dehn-Thurston coordinates are $D T(c)=([0,0], \ldots,[2, t]$, $\ldots,[0,0], 0, \ldots, 0)$ so that $|t| \leq 2$. See figure 2.4 .

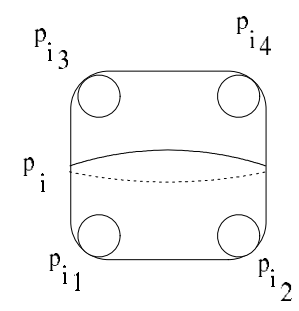

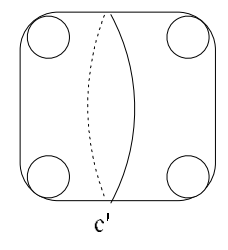

(a)

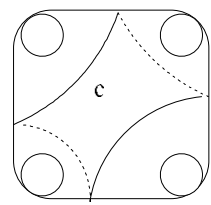

$p_{i}$

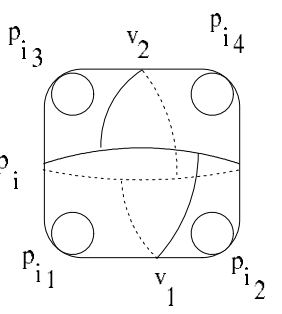

(b)

Figure 2.4 


\section{Fenchel-Nielsen coordinates of Teichmüller space}

In this section, we will recall the definition of the Fenchel-Nielsen coordinates on Teichmüller space. The definition below is tailored to our purposes and differs slightly from the usual one (for instance in [8]), but they are equivalent. The basic setup for the Fenchel-Nielsen coordinates is a surface with a colored hexagonal decomposition. The difficulty in defining the coordinates is due to the change in the underlying surfaces as the metric varies in Teichmüller space.

\subsection{Marked surfaces}

Recall that a marking on an oriented surface $F$ is colored hexagonal decomposition $m=(p, b, c o l)$ of the surface. A marked surface is a pair $(F, m)$ where $m$ is a marking. Two marked surfaces $(F, m)$ and $\left(F^{\prime}, m^{\prime}\right)$ are equivalent if there is an orientation preserving homeomorphism $h: F \rightarrow F^{\prime}$ so that $h(m)$ is isotopic to $m^{\prime}$. It is clear from the definition that a self-homeomorphism $h: F \rightarrow F$ is isotopic to the identity if and only if $h(m)$ is isotopic to $m$. A marked hyperbolic surface is a triple $(F, m, d)$ where $(F, m)$ is a marked surface and $d$ is a hyperbolic metric on $F$ with geodesic boundaries. Two marked hyperbolic surfaces $(F, m, d)$ and $\left(F^{\prime}, m^{\prime}, d^{\prime}\right)$ are equivalent if there is an orientation preserving isometry $h: F \rightarrow F^{\prime}$ so that $h(m)$ is isotopic to $m^{\prime}$.

Fix a marked surface $\left(F, m_{0}\right)$. The Teichmüller space of the marked surface, denoted by $T(F)$ is the space of all equivalence classes of marked hyperbolic surface $(G, m, d)$ so that $(G, m)$ is equivalent to $\left(F, m_{0}\right)$.

\subsection{Metric twisting}

To define the Fenchel-Nielsen coordinate, we will first need the following well known lemma. See [4] (lemma 1.7.1) for a proof.

Lemma 3.1 Let $F_{0,3}$ be the 3-holed sphere with boundary components $\partial_{1}, \partial_{2}, \partial_{3}$.

(a) For any three positive real numbers $x_{1}, x_{2}, x_{3}$, there exists a hyperbolic metric $d$ on $F_{0,3}$ so that the boundary components $\partial_{i}$ are geodesics of lengths $x_{i}$. Furthermore, the metric $d$ is unique up to isometry.

(b) If the distinct pairs of geodesic boundary components in (a) are joined by the shortest geodesic arcs, then these three arcs are disjoint and cut the surface into two isometric right-angled hexagons. 
We also need to introduce the notion of "metric twisting of a marked Riemannian annulus along a geodesic" in order to define the coordinate. Let $A=[-1,1] \times S^{1}$ be an oriented annulus with a Riemannian metric $d$ so that the curve $\{0\} \times S^{1}$ is a geodesic. A marking on $A$ is the homotopy (rel endpoints) class of a path $a:[-1,1] \rightarrow A$ so that $a( \pm 1) \in\{ \pm 1\} \times S^{1}$. Fix a real number $t$. The metric $t$-twisting of a marked Riemannian annulus $(A,[a], d)$ is a new marked Riemannian annulus $\left(A^{\prime},\left[a^{\prime}\right], d^{\prime}\right)$ defined as follows. First cut the annulus $A$ open along the geodesic $\{0\} \times S^{1}$ to obtain two annuli $A_{-}=[-1,0] \times S^{1}$ and $A_{+}=[0,1] \times S^{1}$. Let $S_{ \pm}^{1}$ be the geodesic boundary of $A_{ \pm}$corresponding to $\{0\} \times S^{1}$ and let $\phi: S_{-} \rightarrow S_{+}$be the isometry so that $A=A_{+} \cup_{\phi} A_{-}$. The circles $S_{ \pm}^{1}$ have the induced orientations from $A_{ \pm}$and $\phi$ is orientation reversing. Let $\psi:\left\{R e^{i \theta} \mid \theta \in \mathbb{R}\right\} \rightarrow S_{+}^{1}$ be an orientation preserving isometry and $\rho: S_{+}^{1} \rightarrow S_{+}^{1}$ be the $t$-twisting of $S_{+}^{1}$ which sends $x$ to $\psi\left(e^{2 \pi i t} \psi^{-1}(x)\right)$. Define the new annuli $A^{\prime}$ to be $A_{+} \cup_{\rho \phi} A_{-}$. The Riemannian metric $d^{\prime}$ on $A^{\prime}$ is the gluing metric. To define the marking, let us represent the original marking $[a]$ by a path $a$ so that $a(0)=a([-1,1]) \cap\left(\{0\} \times S^{1}\right)$. The new path $a^{\prime}$ on $A^{\prime}$ is given by $\left[\left.a\right|_{[-1,0]}\right] *[b] *\left[\left.a\right|_{[0,1]}\right]$ where $[x]$ denotes the image of $x$ under the quotient map $A_{+} \cup A_{-} \rightarrow A^{\prime}, *$ denotes the multiplication of paths, and $b$ is the geodesic path of length $|t|$ in $S_{+}^{1}$ starting from $\rho(\phi(a(0)))$ and ending at $a(0)$ so that the orientation of $b$ coincides with that of $S_{+}^{1}$ if and only if $t>0$. Note that there is a natural identification of the boundary of $A$ and $A^{\prime}$. For simplicity, we will assume that $\partial A=\partial A^{\prime}$ under this identification. There exists an orientation preserving homeomorphism $h: A \rightarrow A^{\prime}$ so that $\left.h\right|_{\partial A}=i d$ and $h(a)$ and $a^{\prime}$ are homotopic rel endpoints. Thus the marked annuli $(A,[a])$ and $\left(A^{\prime},\left[a^{\prime}\right]\right)$ are equivalent. For simplicity, we will denote $\left(A^{\prime},\left[a^{\prime}\right], d^{\prime}\right)$ by $T_{t}(A,[a], d),\left[a^{\prime}\right]=T_{t}([a])$, and $d^{\prime}=T_{t}(d)$.

One can also simplify the marking somewhat as follows. It is well known that each path $a:[-1,1] \rightarrow[-1,1] \times S^{1}$ with $a( \pm 1) \in\{ \pm 1\} \times S^{1}$ is relative homotopic to an embedded arc. Also relative homotopic embedded arcs are isotopic by isotopies fixing the endpoints. Thus each marking $[a]$ corresponds to a unique isotopy class of proper arc. For this reason, we will usually represent the marking by the isotopy class.

It follows from the definition that the following holds.

Lemma 3.2 If $t_{1}, t_{2} \in \mathbb{R}$, then $T_{t_{1}}\left(T_{t_{2}}(A,[a], d)\right)$ is isometric to $T_{t_{1}+t_{2}}(A,[a], d)$ by an orientation preserving isometry preserving the marking.

3.3 We now recall the Fenchel-Nielsen coordinates on the Teichmüller space $T(F)$ of a marked surface $(F, m)$. Let $N=3 g+r-3$. Given a point $x=$ $\left(x_{1}, t_{1}, x_{2}, t_{3}, \ldots ., x_{N}, t_{N}, x_{N+1}, \ldots, x_{N+r}\right) \in\left(\mathbb{R}_{>0} \times \mathbb{R}\right)^{N} \times \mathbb{R}_{>0}$, we will describe the corresponding hyperbolic metric $(F N)^{-1}(x)=[d] \in T(F)$ as follows. 
Suppose the marking $m$ is $(p, b, c o l)$ where $p=p_{1} \cup \ldots \cup p_{3 g+r-3}$ and $p_{3 g+r-3+i}$ is the $i$-th boundary component of $F$. Suppose $P$ is a component of $F-p_{1} \cup$ $\ldots \cup p_{3 g+r-3}$ bounded by $p_{i}, p_{k}$ and $p_{l}$ so that the cyclic order $i \rightarrow k \rightarrow l \rightarrow i$ coincides with the cyclic orientation on the boundary of its red hexagon. Then we denote this component by $P_{i j k}$. Note that except for the closed surface of genus 2, only one component of the form $P_{i j k}$ or $P_{i k j}$ can exist.

Now give each 3 -holed sphere $P_{i j k}$ a hyperbolic metric so that so that (1) the length of $p_{r}$ is $x_{r}$ and (2) each arc in $b \cap P_{i j k}$ is the shortest geodesic arc perpendicular to the boundary. The red hexagon in $P_{i j k}$ is now represented by a right-angled hexagon $H_{i j k}$.

We construct the hyperbolic surface $(F N)^{-1}(x)$ in two steps. Let $x^{\prime}=\left(x_{1}, 0\right.$, $\left.x_{2}, 0, \ldots, x_{N}, 0, x_{N+1}, \ldots, x_{N+1}\right)$ be the point having the same $x_{i}$-th coordinate as $x$ but zero twisting coordinates. Then the hyperbolic surface in $T(F)$ having Fenchel-Nielsen coordinates $x^{\prime}$ is constructed as follows. Glue $P_{i j k}$ and $P_{i r s}$ along $p_{i}$ by an orientation reversing isometry so that it sends the red interval $p_{i} \cap H_{i j k}$ to the red interval $p_{i} \cap H_{\text {irs }}$. This gluing produces a new hyperbolic surface $\left(F^{\prime}, d^{\prime}\right)$ homeomorphic to $F$. The marking $m^{\prime}=\left(p_{1}^{\prime} \cup \ldots \cup p_{3 g+r-3}^{\prime}, b^{\prime}, c o l^{\prime}\right)$ on $F^{\prime}$ comes from the quotient of $\cup p_{i}$ and $\cup\left(b \cap P_{i j k}\right)$ and the red hexagons $H_{i j k}$. By the construction, the marked surfaces $(F, m)$ and $\left(F^{\prime}, m^{\prime}\right)$ are equivalent. This gives the point $(F N)^{-1}\left(x^{\prime}\right) \in T(F)$.

For a general point $x \in\left(\mathbb{R}_{>0} \times \mathbb{R}\right)^{3 g+r-3} \times \mathbb{R}_{>0}$, the underlying hyperbolic surface $F^{\prime \prime}$ having $x$ as its Fenchel-Nielsen coordinates is obtained from $F^{\prime}$ by performing metric $t_{i}$ twisting on each Riemannian annulus $N\left(p_{i}\right)$ along the geodesic $p_{i}$. The marking $m^{\prime \prime}=\left(p^{\prime \prime}, b^{\prime \prime}, c o l^{\prime \prime}\right)$ on $F^{\prime \prime}$ is defined as follows. The 3-holed sphere decomposition of $F^{\prime \prime}$ corresponds to the quotient of $\cup_{i} p_{i}$ in $\cup P_{i j k}$. To find the hexagonal decomposition, choose the marking $m^{\prime}=$ $\left(p^{\prime}, b^{\prime}, c o l^{\prime}\right)$ on $F^{\prime}$ so that $b^{\prime} \cap N\left(p_{i}^{\prime}\right)$ consists of two arcs $c_{i_{1}}, c_{i_{2}}$. Now each isotopy class $\left[c_{i_{r}}\right]$ in the annulus $N\left(p_{i}^{\prime}\right)$ is a marking. The new isotopy class of $\operatorname{arcs} T_{t_{i}}\left(\left[c_{i_{r}}\right]\right)$ is represented by an embedded arc $c_{i_{r}}^{\prime}$ having the same endpoints as that of $c_{i_{r}}$. We defines $b^{\prime \prime}$ to be the quotient of $\left(b-\cup_{i} i n t\left(N\left(p_{i}\right)\right)\right) \cup\left(\cup_{i, r} c_{i_{r}}^{\prime}\right)$. Define the coloring of the hexagons in $F^{\prime \prime}-p^{\prime \prime} \cup b^{\prime \prime}$ by the corresponding coloring of $F^{\prime}$. By the construction, we see that the marked surface $\left(F^{\prime \prime}, m^{\prime \prime}\right)$ is equivalent to $(F, m)$. This gives the full description of the Fenchel-Nielsen coordinate.

The use of the marking is to identify the homotopy classes of loops and elements in $C S(F)$ on different surfaces. To be more precise, consider the two marked surfaces $\left(F^{\prime}, m^{\prime}\right)$ and $\left(F^{\prime \prime}, m^{\prime \prime}\right)$ constructed above. By the construction, there is an orientation preserving homeomorphism $h: F^{\prime} \rightarrow F^{\prime \prime}$ so that $h\left(m^{\prime}\right)$ is isotopic to $m^{\prime \prime}$. This homeomorphism induces a bijection between $C S\left(F^{\prime}\right)$ and 
$C S\left(F^{\prime \prime}\right)$ as follows. If $a^{\prime}$ is a curve system in $F^{\prime}$, then the corresponding curve system $a^{\prime \prime}$ homotopic to $h\left(a^{\prime}\right)$ is obtained in the following procedure. Cut $a^{\prime}$ open along all $p_{i}$ 's to obtain a collection of geodesic arcs in $P_{i j k}$. Now rejoin these arcs at the ends points in pairs according to the original cutting points by the oriented geodesic arcs in $p_{i}$ of length $x_{i}\left|t_{i}\right|$ from the left side endpoints to the right side endpoints along $p_{i}$. The resulting curve system is $a^{\prime \prime}$. It follows from the construction that,

$$
l_{d^{\prime \prime}}\left(\left[a^{\prime \prime}\right]\right) \leq l_{d^{\prime}}\left(\left[a^{\prime}\right]\right)+\sum_{i=1}^{3 g+r-3} x_{i}\left|t_{i}\right| I\left([a], p_{i}\right) .
$$

The basic result about the Fenchel-Nielsen coordinates is that the map $F N$ : $T(F) \rightarrow\left(\mathbb{R}_{>0} \times \mathbb{R}\right)^{3 g+r-3} \times \mathbb{R}_{>0}$ is a homeomorphism. See for instance [8] chapter 8 , or [4] chapter 6 .

\section{Proof of the main theorem}

We prove the main theorem in this section. There are two facts about hyperbolic polygons used in the proof. These two facts will be established in section 5 . In subsections 4.1-4.4, we prove the first inequality (1.1). In the remaining subsections, we establish (1.2).

To begin the proof, we fix a marking on the surface and let $F N$ and $D T$ be the associated coordinates on the Teichmüller space $T(F)$ and the space of curve systems $C S(F)$.

4.1 To prove inequality (1.1) for all metrics $[d] \in T(F)$ and $[a],[b] \in C S(F)$, by the remarks in subsection 2.3 , it suffices to show

$$
\left|l_{d}([a])-l_{d}([b])\right| \leq 6|F N(d)|
$$

whenever $D T(a)$ and $D T(b)$ differ only in one intersection coordinate $x_{i}$ by 2 , ie, $x_{i}(a)=x_{i}(b)+2$ and $x_{j}(a)=x_{j}(b)$ for all $j \neq i$ and $t_{k}(a)=t_{k}(b)$ for all $k$. There are three subcases we have to consider according to the nature of the decomposing loop $p_{i}:(1)\left[p_{i}\right] \in C S(F)$ and is adjacent to only one 3-holed sphere $P_{i i j} ;(2)\left[p_{i}\right] \in C S(F)$ and is adjacent to two different 3 -holed spheres $P_{i i_{1} i_{2}}$ and $P_{i i_{3} i_{4}} ;(3) p_{i} \subset \partial F$. 
4.2 In the first case, by proposition 2.3 , we can write $a \cong p_{j}^{e_{1}} c^{e_{2}} b$ where $e_{1}, e_{2} \in\{0, \pm 1, \pm 2\}$ and $c$ is as shown in figure 2.3.

We can write the loop $c \cong p_{i}^{ \pm 1} c^{\prime}$ where $c^{\prime}$ has zero twisting coordinates as shown in figure 2.3. Let $l(S)$ be the length of the shortest geodesic segment in the 3-holed sphere $P_{i i j}$ joining the two boundary components corresponding to $p_{i}$. Then by the definition of the Fenchel-Nielsen coordinates, we have $l_{d}\left(\left[c^{\prime}\right]\right) \leq x_{i}(d)\left|t_{i}(d)\right|+l(S)$. This shows

$$
\begin{aligned}
\left|l_{d}([a])-l_{d}([b])\right| & \leq l_{d}\left(\left[p_{j}^{e_{1}} c^{e_{2}}\right]\right) \\
& \leq 2 l_{d}\left(\left[p_{j}\right]\right)+2 l_{d}([c]) \\
& \leq 2 x_{j}(d)+2 l_{d}\left(\left[p_{i}^{ \pm 1} c^{\prime}\right]\right) \\
& \leq 2 x_{j}(d)+2 x_{i}(d)+2 l_{d}\left(\left[c^{\prime}\right]\right) \\
& \leq 2 x_{j}(d)+2 x_{i}(d)+2 x_{i}(d)\left|t_{i}(d)\right|+2 l(S) .
\end{aligned}
$$

By proposition 5.1, we can estimate the length $l(S)$ in terms of the red rightangled hexagon inside $P_{i i j}$. Thus we obtain,

$$
l(S) \leq 2 / x_{i}(d)+2 / x_{j}(d)+x_{j}(d) / 2+2 \log 2 .
$$

Combining these together, we obtain

$$
\begin{aligned}
\left|l_{d}([a])-l_{d}([b])\right| & \leq 4 x_{j}(d)+2 x_{i}(d)+4 / x_{j}(d)+4 / x_{i}(d)+2 x_{i}(d)\left|t_{i}(d)\right|+4 \log 2 \\
& \leq 4|F N(d)| \\
& \leq 2|F N(d)||D T(a)-D T(b)| .
\end{aligned}
$$

4.3 In the second case, we use proposition 2.4. Thus $a \cong p_{i_{1}}^{s_{1}} \ldots p_{i_{4}}^{s_{4}} c^{e} b$ where $\left|s_{1}\right|+\left|s_{2}\right| \leq 2,\left|s_{3}\right|+\left|s_{4}\right| \leq 2, e \in\{ \pm 1\}$ and $c$ has Dehn-Thurston coordinates of the form $([0,0], \ldots,[0,0],[2, t],[0,0], \ldots, 0)$ where $|t| \leq 2$. See figure 2.4. By the triangle inequality,

$$
\left|l_{d}([a])-l_{d}([b])\right| \leq 2 \sum_{j=1}^{4} l_{d}\left(\left[p_{i_{j}}\right]\right)+l_{d}([c]) .
$$

To estimate $c$, let $c^{\prime} \cong c_{z t}$. Then $c \cong p_{i}^{t} c^{\prime}$ where $|t| \leq 2$ hence $l_{d}([c]) \leq$ $l_{d}\left(\left[c^{\prime}\right]\right)+2 x_{i}(d)$.

Consider the metric $d^{\prime}$ on $F$ so that $F N(d)$ and $F N\left(d^{\prime}\right)$ are the same except at the $i$-th twisting coordinate where $t_{i}\left(d^{\prime}\right)=0$. Then by the definition of the Fenchel-Nielsen coordinate $l_{d}\left(\left[c^{\prime}\right]\right) \leq l_{d^{\prime}}\left(\left[c^{\prime}\right]\right)+2 x_{i}(d)\left|t_{i}(d)\right|$. We will estimate the length $l_{d^{\prime}}\left(\left[c^{\prime}\right]\right)$ as follows. Let $v_{1}$ and $v_{2}$ be the shortest arcs in the redhexagons $H_{i i_{1} i_{2}}$ and $H_{i i_{3} i_{4}}$ joining the $p_{i}$-side to its opposite side (see figure 2.4(b)). Then by the construction of the Fenchel-Nielsen coordinates, we have 
$l_{d^{\prime}}\left(\left[c^{\prime}\right]\right)=l_{d^{\prime}}\left(v_{1}\right)+l_{d^{\prime}}\left(v_{2}\right)$. By proposition 5.1, we can estimate the lengths $l_{d^{\prime}}\left(v_{k}\right)$ for $k=1,2$ as follows. For simplicity, we write $x_{r}=x_{r}(d)$.

$$
\begin{aligned}
& l_{d^{\prime}}\left(v_{1}\right) \leq 2 / x_{i}+2 / x_{i_{1}}+x_{i_{1}} / 2+x_{i_{2}} / 2+\log 2 . \\
& l_{d^{\prime}}\left(v_{2}\right) \leq 2 / x_{i}+2 / x_{i_{3}}+x_{i_{3}} / 2+x_{i_{4}} / 2+\log 2 .
\end{aligned}
$$

Combining the above formulas, we obtain

$$
\begin{aligned}
& \left|l_{d}([a])-l_{d}([b])\right| \\
& \quad \leq 2 \sum_{j=1}^{4} x_{i_{j}}+2 x_{i}+x_{i}(d)\left|t_{i}(d)\right|+4 / x_{i}+2 / x_{i_{1}}+2 / x_{i_{3}}+x_{i_{1}}+ \\
& \quad \leq 6|F N(d)| \\
& \quad \leq 3|F N(d)||D T(a)-D T(b)| .
\end{aligned}
$$

Note the coefficient is 6 instead of 4 since $i_{1}, i_{2}, i_{3}$, and $i_{4}$ need not be distinct indices.

4.4 In the third case that $x_{i}(a)=x_{i}(b)+2$ where $p_{i} \subset \partial F$, the result follows from the previous case by the standard metric double construction. Indeed, let $F^{*}$ be the double of $F$ across its boundary, ie, $F^{*}=F \cup_{i d} F$ where $i d$ is the identity map on $\partial F$. We give $F^{*}$ the double metric $d^{*}$ and the marking the double of the original marking. The double of a curve system $\alpha \in C S(F)$ is denoted by $\alpha^{*} \in C S\left(F^{*}\right)$. Note that the twisting coordinate of $\alpha^{*}$ at each boundary component is always zero. Then it follows from the definition that $\left|F N\left(d^{*}\right)\right| \leq 2|F N(d)|$, and $\left|D T\left([a]^{*}\right)-D T\left([b]^{*}\right)\right|=2$. Thus by the boundaryless case,

$$
\begin{gathered}
\left|l_{d}([a])-l_{d}([b])\right|=1 / 2\left|l_{d^{*}}\left([a]^{*}\right)-l_{d^{*}}\left([b]^{*}\right)\right| \leq 3\left|F N\left(d^{*}\right)\right| \\
\leq 6|F N(d)|=3|F N(d)||D T(a)-D T(b)| .
\end{gathered}
$$

4.5 To prove the second inequality (1.2), we first consider the two cases $F N\left(d_{1}\right)-F N\left(d_{2}\right)=(0, \ldots, 0, c, 0, \ldots 0) \in\left(\mathbb{R}_{>0} \times \mathbb{R}\right)^{N} \times R_{>0}^{r}$ where either $c$ is $t_{i}\left(d_{1}\right)-t_{i}\left(d_{2}\right)$ or is $x_{j}\left(d_{1}\right)-x_{j}\left(d_{2}\right)$. The general case follows by a simple interpolation. These two cases will be dealt separately.

4.6 In the first case that $c=t_{i}\left(d_{1}\right)-t_{i}\left(d_{2}\right)$, then the metric $d_{2}$ is obtained from $d_{1}$ by a metric twisting of signed length $x_{i}\left(d_{1}\right) c$. Thus if $a \in \alpha$ is a $d_{1}$-geodesic representative, then a representative $a^{\prime} \in \alpha$ in the $d_{2}$-surface is obtained from $a$ by cutting $a$ open along $p_{i}$ and gluing $I\left(\alpha, p_{i}\right)$ many copies of geodesic segments of lengths $x_{i}\left(d_{1}\right)|c|$ as obtained in the inequality (3.1). Thus

$$
\left|l_{d_{1}}(\alpha)-l_{d_{2}}(\alpha)\right| \leq x_{i}\left(d_{1}\right)|c||D T(\alpha)| \leq D\left(F N\left(d_{1}\right), F N\left(d_{2}\right)\right)|D T(\alpha)| .
$$


4.7 In the second case that $c=x_{i}\left(d_{1}\right)-x_{i}\left(d_{2}\right)$, due to symmetry, it suffices to show that

$$
l_{d_{2}}(\alpha) \leq l_{d_{1}}(\alpha)+4 D\left(F N\left(d_{1}\right), F N\left(d_{2}\right)\right)|D T(\alpha)| .
$$

To this end, take a $d_{1}$-geodesic representative $a \in \alpha$. We will construct a piecewise geodesic representative $a^{\prime} \in \alpha$ in $d_{2}$-surface and estimate the length $l_{d_{2}}\left(a^{\prime}\right)$. The $d_{2}$-surface $F^{\prime}$ is obtained from the $d_{1}$-surface by cutting open along the geodesic $p_{i}$. Then replace the 3 -holed spheres $P_{i j k}$ and $P_{i r s}$ adjacent to $p_{i}$ by new pairs so that the lengths at $p_{i}$ are $l_{d_{2}}\left(\left[p_{i}\right]\right)$, and all other lengths remain the same. For each 3 -holed sphere $P$ in the decomposition, let $H$ in $P$ be one of the right-angled hexagon obtained from lemma 3.1(b). Note that the metric gluing to obtain the $d_{2}$-surface has the same twisting angles $t_{j}$. This shows that there is an orientation preserving homeomorphism $h$ from the $d_{1}$-surface to the $d_{2}$-surface so that (1) $h$ sends the right-angled-hexagon $H$ to the right-angled-hexagon $H$; (2) $h$ on each edge in the boundary of the right-angled hexagons $H$ and $P-H$ are homothetic maps. (Note that the redhexagons used as part of a marking on the $d_{k}$-surface are in general different from the hexagons $H$.) The representative $a^{\prime}$ is choosen so that on each rightangled hexagon $X=H$ or $P-H, a^{\prime}$ consists of geodesic segments and for each component $b$ of $a \cap X$, there exists exactly one component $b^{\prime}$ of $a^{\prime} \cap X$ for which $h(\partial b)=\partial b^{\prime}$.

It follows from the construction that $l_{d_{2}}\left(b^{\prime}\right)=l_{d_{1}}(b)$ unless $b$ lies in either $P_{i j k}$ or $P_{\text {irs }}$. In the later case, by theorem 5.2 , we have

$$
l_{d_{2}}\left(b^{\prime}\right) \leq l_{d_{1}}(b)+4\left|\log \sinh \left(x_{i}\left(d_{1}\right) / 2\right)-\log \sinh \left(x_{i}\left(d_{2}\right) / 2\right)\right| .
$$

Let $n$ be sum of the number of components of $a \cap X$ for all right-angled hexagons $X$ in $P_{i j k}$ and $P_{i r s}$. Then

$$
l_{d_{2}}(\alpha) \leq l_{d_{2}}\left(a^{\prime}\right) \leq l_{d_{1}}(\alpha)+4 n\left|\log \sinh \left(x_{i}\left(d_{1}\right) / 2\right)-\log \sinh \left(x_{i}\left(d_{2}\right) / 2\right)\right| .
$$

It remains to estimate the number $n$.

Lemma 4.1 Under the above assumptions

$$
n \leq\left(\left|t_{i}\left(d_{1}\right)\right|+\left|t_{j}\left(d_{1}\right)\right|+\left|t_{k}\left(d_{1}\right)\right|+\left|t_{r}\left(d_{1}\right)\right|+\left|t_{s}\left(d_{1}\right)\right|+7\right)|D T(\alpha)| .
$$

Assuming this lemma, then we obtain the required estimate that

$$
\begin{aligned}
l_{d_{2}}(\alpha) & \leq l_{d_{2}}\left(a^{\prime}\right) \\
& \leq l_{d_{1}}(\alpha)+4\left(\left|t_{i}\right|+\left|t_{j}\right|+\left|t_{k}\right|+\left|t_{r}\right|+\left|t_{s}\right|+7\right) \mid \log \sinh \left(x_{i}\left(d_{1}\right) / 2\right) \\
& \leq l_{d_{1}}(\alpha)+4 D\left(F N\left(d_{1}\right), F N\left(d_{2}\right)\right)|D T(\alpha)|
\end{aligned}
$$


where $t_{n}=t_{n}\left(d_{1}\right)$. Thus the inequality (1.2) follows in this case.

Proof of lemma 4.1 Let us first consider the special case that $t_{j}\left(d_{1}\right)=0$ for all $j$. In this case the red-hexagons in the $d_{1}$-surface are the same as the right-angled hexagon $H$. Thus $n \leq I(\alpha, p)+I(\alpha, b)$ where $(p, b, c o l)$ is the marking on the $d_{1}$-surface. Now we can write $\alpha=\left[p_{1}^{r_{1}} \ldots . p_{N}^{r_{N}}\right] \alpha_{z t}$ where $r_{i}$ is the Dehn-Thurston twisting coordinate of $\alpha$ and $\alpha_{z t}$ has zero twisting coordinates. Thus,

$$
\begin{aligned}
n & \leq I(\alpha, p)+I\left(\alpha_{z t}, b\right)+I\left(p_{1}^{\left|r_{1}\right|} \ldots p_{N}^{\left|r_{N}\right|}, b\right) \\
& \leq 2 I(\alpha, p)+2 \sum_{i=1}^{N}\left|r_{i}\right| \\
& \leq 2|D T(\alpha)| .
\end{aligned}
$$

In particular, the conclusion holds in this case. Also we see that for any marking $(p, b, c o l)$ on a surface, $I(\alpha, p)+I(\alpha, b) \leq 2|D T(\alpha)|$.

In the general case that some $t_{j}\left(d_{1}\right) \neq 0$, we take all $p_{j}$ 's to be $d_{1}$-geodesics and let $u_{h l}$ be the shortest geodesic segment joining $p_{h}$ to $p_{l}$ when $p_{h}$ and $p_{l}$ lie inside some 3-holed sphere component of $F-p$. Let $b$ be the $d_{1}$-geodesic representative of the marking curve and $b_{h l}$ be the component of $b \cap P_{h l m}$ corresponding to $u_{h l}$. Then by definition of Fenchel-Nielson coordinates, $u_{h l}$ is relatively homotopic to $w_{h} * b_{h l} * w_{l}$ where $w_{h}$ is a geodesic path in $p_{h}$ of length $x_{h}\left(d_{1}\right)\left|t_{h}\left(d_{1}\right)\right|$. Thus the number of new intersection points in $a \cap w_{h}$ is at most $\left(\left|t_{h}\left(d_{1}\right)\right|+1\right) I\left(\alpha, p_{h}\right)$. This shows that

$$
\begin{aligned}
n & \leq|a \cap p|+\sum_{h, l}\left|a \cap\left(\cup_{h, l} u_{h l}\right)\right| \\
& \leq I(\alpha, p)+|a \cap b|+\sum_{h}\left(\left|t_{h}\left(d_{1}\right)\right|+1\right) I\left(\alpha, p_{h}\right) \\
& \leq I(\alpha, p)+I(\alpha, b)+\sum_{h}\left(\left|t_{h}\left(d_{1}\right)\right|+1\right) I(\alpha, p) \\
& \leq\left(\sum_{h}\left|t_{h}\left(d_{1}\right)\right|+7\right)|D T(\alpha)|,
\end{aligned}
$$

where the sum is over the set $\{i, j, k, r, s\}$.

4.8 The above estimate works even if the loop $p_{i}$ is a boundary component of the surface $F$. 


\subsection{The general case}

The general case of any two metrics $d_{1}$ and $d_{2}$ follows from interpolation. Namely we use the formula $\left|F\left(x_{i}, t_{i}\right)-F\left(y_{i}, s_{i}\right)\right| \leq\left|F\left(x_{i}, t_{i}\right)-F\left(y_{i}, t_{i}\right)\right|+$ $\left|F\left(y_{i}, t_{i}\right)-F\left(y_{i}, s_{i}\right)\right|$. Thus the result follows. Also the corollary 1.2 follows from the standard argument involving the definition of the space of measured laminations. See [10] section 6 for the proof of the similar result for the intersection pairing.

\section{$5 \quad$ Elementary facts about hyperbolic polygons}

We will prove two facts used in the proof of the main theorem in this section. For basic information on hyperbolic hexagons, see [1] section 7.19, [4] section 2.4. Suppose $H$ is a right-angled hyperbolic hexagon whose side lengths (reading from counterclockwise) are : $a, z, x, y, b$ and $w$. See figure 1.1.

Proposition 5.1 Consider the right-angled hexagon $H$ above. Let $h$ be the length of the shortest geodesic arc from the $a$-side to the $y$-side. Then:

(a) $w \leq 1 / a+1 / b+x+2 \log 2$.

(b) $h \leq 1 / a+1 / b+b+x+\log 2$ and $h \leq 1 / a+1 / 2(1 / b+1 / x)+b+x+2 \log 2$.

Proof By the cosine rule, $\cosh w=(\cosh x+\cosh a \cosh b) /(\sinh a \sinh b)$. Using $\cosh a \cosh b+\cosh c \leq \cosh a \cosh b(\cosh c+1)$ and $\cosh w \geq 1 / 2 e^{w}$, we obtain

$$
1 / 2 e^{w} \leq \operatorname{coth} a \operatorname{coth} b(\cosh x+1) .
$$

Taking logs, we get

$$
w-\log 2 \leq \log \operatorname{coth} a+\log \operatorname{coth} b+\log (\cosh x+1) .
$$

On the other hand, $\operatorname{coth} a \leq 1+1 / a$. Thus $\log \operatorname{coth} a \leq \log (1+1 / a) \leq 1 / a$. Similarly, $\log \operatorname{coth} b \leq 1 / b$. Finally, $\log (\cosh x+1) \leq \log \left(e^{x}+1\right) \leq x+\log 2$. Put all these together, we obtain the estimate (a).

To see (b), by the cosine law for pentagon,

$$
\cosh h=\sinh b \sinh w .
$$

Now $e^{h} / 2 \leq \cosh h$ and $\sinh x \leq e^{x} / 2$. Thus $e^{h} \leq 1 / 2 e^{b} e^{w}$. This shows that $h \leq b+w-\log 2$. By part (a), we obtain

$$
h \leq 1 / a+1 / b+x+b+2 \log 2 .
$$


Also $h \leq 1 / a+1 / x+x+b+\log 2$. Thus

$$
h \leq 1 / a+1 / 2(1 / b+1 / x)+b+x+\log 2 .
$$

Let $A_{g}=A_{g}(\lambda, \mu)$ be a geodesic segment in $H$ joining two sides of $H$ so that the endpoints of $A_{g}$ cut the sides into two intervals of lengths $\lambda t,(1-\lambda) t$ and $\mu r,(1-\mu) r$. In the discussion below, the numbers $a, b, \lambda, \mu$ remain constant. The variable is $x$ and $y, z, w$ depend on $x$. Let $S=S_{\lambda, \mu}$ be the length of $A_{g}$. Our goal is to estimate the rate of change of $S_{\lambda, \mu}$ with respect to $x$.

Theorem 5.2 Under the above assumption, we have $\left|\frac{d S}{d x}\right| \leq 4 \operatorname{coth} x$.

Proof We begin with several simple lemmas based on the cosine and sine laws in hyperbolic geometry.

Lemma 5.3 In the right-angled hexagon $H$,

(a) $\frac{d y}{d x}=-\frac{\operatorname{coth} z}{\sinh x}$.

(b) $\frac{d w}{d x}=\frac{1}{\sinh a \sinh z}$.

(c) $\left|\frac{d y}{d x}\right|<\operatorname{coth} x$ and $\frac{d y}{d x}<0$.

(d) $0<\frac{d w}{d x}<1$.

(e) $\cosh x>\operatorname{coth} z$ and $\sinh x \sinh z>1$.

Proof From the cosine rule: $\cosh x=(\cosh w+\cosh y \cosh z) /(\sinh y \sinh z)=$ $\cosh w /(\sinh y \sinh z)+\operatorname{coth} y \operatorname{coth} z>\operatorname{coth} y \operatorname{coth} z>\operatorname{coth} z$. Now squaring the inequality and using $\cosh ^{2} x=1+\sinh ^{2} x$ and $\operatorname{coth}^{2} z=1+1 / \sinh ^{2} z$, we obtain $\sinh x \sinh z>1$. This shows $(e)$.

Differentiating the other cosine rule

$$
\cosh y=(\cosh a+\cosh b \cosh x) /(\sinh b \sinh x)
$$

gives $\frac{d y}{d x}=-(\cosh b+\cosh a \cosh x) /\left(\sinh b \sinh ^{2} x \sinh y\right)$. Plugging in the cosine rule $\cosh b+\cosh a \cosh x=\sinh a \sinh x \cosh z$, we obtain,

$$
\begin{aligned}
\frac{d y}{d x} & =-(\sinh a \sinh x \cosh z) /\left(\sinh b \sinh ^{2} x \sinh y\right) \\
& =-(\sinh a \cosh z) /(\sinh b \sinh x \sinh y) .
\end{aligned}
$$

Plugging in the sine rule $\sinh a / \sinh y=\sinh b / \sinh z$ gives

$$
\frac{d y}{d x}=-\cosh z /(\sinh x \sinh z)=-\operatorname{coth} z / \sinh x .
$$


This shows (a) and the second part of (c).

By the inequality (e) above $\left|\frac{d y}{d x}\right|<\operatorname{coth} x / \operatorname{coth} y<\operatorname{coth} x$. This shows (c).

For $\frac{d w}{d x}$, we have $\cosh w=(\cosh x+\cosh a \cosh b) /(\sinh a \sinh b)$. By the sine law,

$$
\frac{d w}{d x}=\sinh x /(\sinh a \sinh b \sinh w)=1 /(\sinh b \sinh y)=1 /(\sinh a \sinh z) .
$$

By the rewritten form of (e) for the pair $(a, z)$ instead of $(z, x)$, we have $\sinh a \sinh z>1$. This shows $0<\frac{d w}{d x}<1$. Thus both (b) and (d) hold.

The next lemma is well known. It is a simple application of the sine law. We will omit the details of the proof.

Lemma 5.4 Suppose $\Delta q p r$ is a hyperbolic triangle with angle at $p$ being $\alpha$. Suppose starting at time $t=0$ the endpoint $p$ moves along the ray $p r$ with unit speed while the other two points $q, r$ remain fixed. Let $l_{p q}$ denote the length between $p$ and $q$. Then $d l_{p q} /\left.d t\right|_{t=0}=-\cos \alpha$.

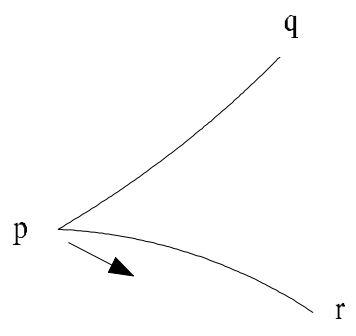

Figure 5.1

The next lemma is crucial for most of the estimates in the proof of theorem 5.2.

Lemma 5.5 Consider a hyperbolic quadrilateral with side lengths and angles (reading from counterclockwise) as $c$ (side), right angle, $t$ (side), right-angle, $e$ (side), $\beta$ (angle), $S$ (side) and $\alpha$ (angle). Consider varying $t$ and holding $c$ and $e$ fixed, then

$$
0<\frac{\partial S}{\partial t}<\operatorname{coth}(t / 2)
$$


Proof By the cosine law, $\cosh S=-\sinh c \sinh e+\cosh c \cosh e \cosh t$. Differentiating this equation gives $\partial S / \partial t=\cosh c \cosh e \sinh t / \sinh S>0$. Plugging in the identity $\cosh v=2 \sinh ^{2}(v / 2)+1$ three times to the above cosine law gives $\sinh ^{2}(S / 2)=\sinh ^{2}((c-e) / 2)+\cosh c \cosh e \sinh ^{2}(t / 2)>\cosh c \cosh e \sinh ^{2}(t / 2)$. Using $\sinh t=2 \operatorname{coth}(t / 2) \sinh ^{2}(t / 2)$, we obtain the result.

We now begin the proof of the theorem 5.2. We will break it into three cases, each of which will have several subcases. We refer to the case where the geodesic segment $A_{g}$ has endpoints on adjacent sides as case 1, sides two apart as case 2 and endpoints on opposite sides as case 3 . In the following discussion, we will assume the hexagon has side lengths $x, y(x), b, w(x), a, z(x)$ where a and $\mathrm{b}$ are fixed. We will use $\frac{d y}{d x}$ etc, for derivatives of these side lengths. When looking at $S(x)$ however, we will often consider $S$ as a side of a hyperbolic polygon with the angles not incident on $S$ all right angles. In such a case, we can vary the other sides independently and we will use $\partial S / \partial c$ for the change in $S$ when we vary only the side $c$ of this polygon.

Case 1 There are up to symmetry three subcases depending on which sides $S$ joins, however we will do all three cases simultaneously with a little care. In this case consider the right-angled triangle cut out by the segment $A_{g}(\lambda, \mu)$. The side lengths of the triangle are $\mu c, \lambda e$ and $S$, where $(c, e)$ may be $(x, y),(y, b)$ or $(b, w)$. Let $\alpha$ be the angle opposite $\mu c$ and $\beta$ the angle opposite $\lambda e$. By lemma 5.4, if $c$ increases one endpoint $S$ moves off at an angle of $\pi-\beta$, hence $\partial S / \partial c=\mu \cos (\beta)$, similarly as $e$ increases the other endpoint of $S$ moves off at an angle of $\pi-\alpha$, hence $\partial S / \partial e=\lambda \cos (\alpha)$. Thus $d S / d x=(\partial S / \partial c)(d c / d x)+$ $(\partial S) /(\partial e)(d e / d x)=\mu \cos (\beta)(d c / d x)+\lambda \cos (\alpha)(d e / d x)$. Since $0<\alpha, \beta<\pi / 2$, the cosines are positive. In any of the three cases for $(c, e)$, by lemma 5.3 , we have $(d c / d x)(d e / d x) \leq 0$. Therefore, by lemma 5.3 again,

$$
\begin{aligned}
|d S / d x| & \leq \max (\mu \cos (\beta)|d c / d x|, \lambda \cos (\alpha)|d e / d x|) \\
& \leq \max (|d c / d x|,|d e / d x|)<\operatorname{coth} x
\end{aligned}
$$

Case 2 This case splits into four subcases up to symmetry. We will at least start these cases together. We have a quadrilateral with sides and angles (reading from counterclockwise) as $\mu c$ (side), right-angle, $t$ (side), right-angle, $\lambda e$ (side), $\beta$ (angle), $S$ (side), and $\alpha$ (angle). Here $(c, t, e)$ is one of $(z, x, y)$, $(x, y, b),(y, b, w)$, or $(b, w, a)$.

By Lemma 5.4, $\partial S / \partial c=\mu \cos (\alpha)$ and $\partial S / \partial e=\lambda \cos (\beta)$. Note that both of these have magnitude at most 1 . Combining this fact with Lemma 5.5, we obtain

$$
|d S / d x|=|(\partial S / \partial c)(d c / d x)+(\partial S / \partial e)(d e / d x)+(\partial S / \partial t)(d t / d x)|
$$



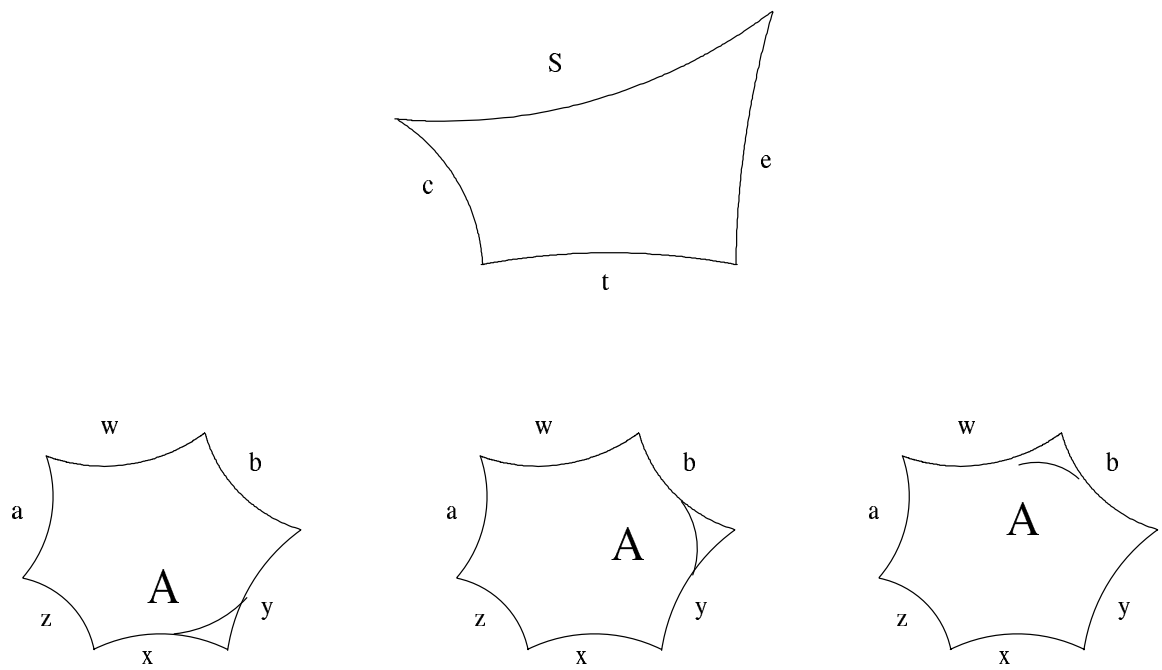

Figure 5.2

$$
\leq|d c / d x|+|d e / d x|+\operatorname{coth}(t / 2)|d t / d x| .
$$

In any case, by lemma 5.3, $|d c / d x|<\operatorname{coth} x$ and $|d e / d x|<\operatorname{coth} x$. Hence $|d S / d x| \leq 2 \operatorname{coth} x+\operatorname{coth}(t / 2)|d t / d x|$.

Subcase (i). $(c, t, e)=(z, x, y)$. In this case $t=x, d t / d x=1$ and using the fact that $2 \operatorname{coth} x=\operatorname{coth}(x / 2)+\tanh (x / 2)>\operatorname{coth}(x / 2)$ we see $|d S / d x|<4 \operatorname{coth} x$.

Subcase (ii). $(c, t, e)=(x, y, b)$. In this case $t=y, d e / d x=0$, and by lemma 5.3 we have

$$
\begin{aligned}
|d S / d x| & \leq|d c / d x|+\operatorname{coth}(t / 2)|d t / d x| \\
& <\operatorname{coth} x+\operatorname{coth}(y / 2) \operatorname{coth} z / \sinh x \\
& <\operatorname{coth} x+\operatorname{coth}(y / 2) \operatorname{coth} x / \operatorname{coth} y<3 \operatorname{coth} x .
\end{aligned}
$$

Note that $\operatorname{coth} z / \sinh x<\operatorname{coth} x / \operatorname{coth} y$ by the proof of lemma 5.3.

Subcase (iii). $(c, t, e)=(y, b, w)$. In this case $t=b$ and $d t / d x=0$.

Subcase (iv). $(c, t, e)=(b, w, a)$. In this case $d c / d x=d e / d x=0, t=w$ and $d t / d x=1 /(\sinh a \sinh z)$. Hence

$$
\begin{aligned}
0 & <d S / d x<\operatorname{coth}(w / 2) /(\sinh a \sinh z) \\
& =(1+\cosh w) /(\sinh w \sinh a \sinh z) \\
& =(1+\cosh w) /(\sinh z \sinh x \sinh y) \\
& <2 \cosh w /(\sinh z \sinh x \sinh y) .
\end{aligned}
$$




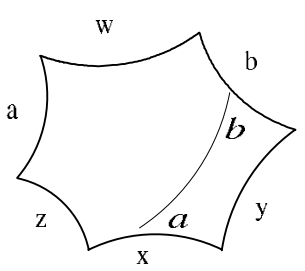

$a$
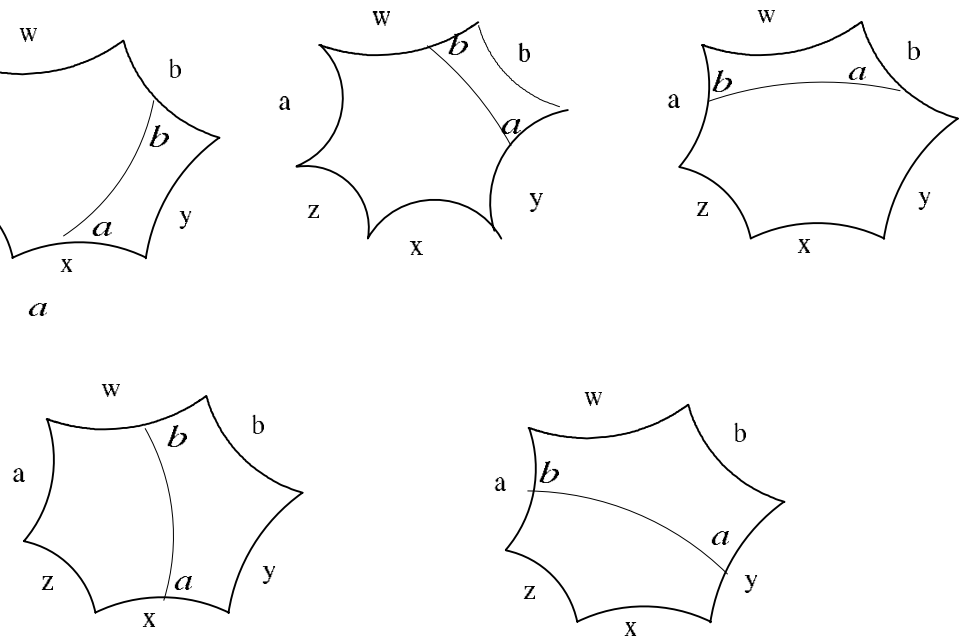

Figure 5.3

Since $\cosh w=\cosh x \sinh y \sinh z-\cosh y \cosh z<\cosh x \sinh y \sinh z$, it follows that $0<d S / d x<2 \operatorname{coth} x$. This completes Case 2 .

Case 3 Here there are two subcases (up to symmetry). Either $S$ joins $x$ to $w$ or $S$ joins $a$ to $y$. In the first subcase we have a pentagon with sides and angles (reading from counterclockwise): $\mu x$ (side), right angle, $y$ (side), right-angle, $b$ (side), right-angle, $\lambda w$ (side), $\beta$ (angle), $S$ (side) and $\alpha$ (angle).

By Lemma 5.4, $\partial S / \partial x=\mu \cos (\alpha)$ hence $|\partial S / \partial x| \leq 1$, and similarly $(\partial S / \partial w)=$ $\lambda \cos (\beta)$ hence $|\partial S / \partial w| \leq 1$. Also from Lemma 5.4, increasing $y$ is equivalent to pulling the endpoint of $S$ off at an angle of $(\pi / 2)+\alpha$ but $\cosh (\mu x)$ times as fast, hence $(\partial S / \partial y)=\cosh (\mu x) \sin (\alpha) \leq \cosh (\mu x)$. Combining these and lemma 5.3, we obtain, $|d S / d x|=\left|(\partial S / \partial x)+(\partial S / \partial w) \frac{d w}{d x}+(\partial S / \partial y) \frac{d y}{d x}\right|$ $\leq 2+\cosh (\mu x) \operatorname{coth} z / \sinh x$. To estimate the size, we note that this case is symmetric. On the other side of $S$ is another pentagon and the same argument gives $|d S / d x| \leq 2+\cosh ((1-\mu) x) \operatorname{coth} y / \sinh x$. Combining these gives

$$
|d S / d x| \leq 2+\min [\cosh (\mu x) \operatorname{coth} z, \cosh ((1-\mu) x) \operatorname{coth} y] / \sinh x .
$$

Since the min is at most the geometric mean we get

$$
|d S / d x| \leq 2+[\cosh (\mu x) \cosh ((1-\mu) x) \operatorname{coth} z \operatorname{coth} y]^{1 / 2} / \sinh x .
$$

By lemma 5.3, coth $y \operatorname{coth} z<\cosh x$ and $\cosh (\mu x) \cosh ((1-\mu) x)=[\cosh (x)+$ $\cosh ((1-2 \mu) x)] / 2<\cosh x$. Hence we get

$$
|d S / d x|<2+\cosh x / \sinh x<3 \operatorname{coth} x .
$$

In the second subcase we have two pentagons. One with sides and angles: $\mu y$ (side), right-angle, $b$ (side), right-angle, $w$ (side), right-angle, $\lambda a$ (side), 
$\beta$ (angle), $S$ (side) and $\alpha$ (angle). The other pentagon has sides and angles: $(1-\lambda) a$ (side), right-angle, $z$ (side), right-angle, $x$ (side), right-angle, $(1-\mu) y$ (side), $\pi-\alpha$ (angle), $S$ (side), $\pi-\beta$ (angle).

Looking at the first pentagon, by Lemma 5.4, $\partial S / \partial y=\mu \cos (\alpha)$ which has magnitude at most 1 . Increasing $w$ by an infinitesimal amount $\delta w$ has the effect of moving an endpoint of $S$ a distance $\cosh (\lambda a) \delta w$ at an angle of $\pi / 2+\beta$. Hence $\partial S / \partial w=-\cos (\pi / 2+\beta) \cosh (\lambda a)$. and $d S / d x=\sin (\beta) \cosh (\lambda a) \frac{d w}{d x}+$ $\mu \cos (\alpha) \frac{d y}{d x}$. Note that the first term in always positive and the second may be either positive or negative. Hence we see that

$$
d S / d x \geq \frac{d y}{d x} \geq-\operatorname{coth} x .
$$

Thus we need only give an upper bound on $d S / d x$. The bound above gives $d S / d x \leq \cosh (\lambda a)\left(\frac{d w}{d x}\right)+\operatorname{coth} x=\cosh (\lambda a) /(\sinh a \sinh z)+\operatorname{coth} x$. We will derive two upper bounds from this. First since

$$
\begin{aligned}
\sinh a & =\cosh (\lambda a) \sinh ((1-\lambda) a)+\sinh (\lambda a) \cosh ((1-\lambda) a) \\
& >\cosh (\lambda a) \sinh ((1-\lambda) a),
\end{aligned}
$$

we have

$$
d S / d x<1 /(\sinh ((1-\lambda) a) \sinh z)+\operatorname{coth} x
$$

Second, since $\cosh (\lambda a) \leq \cosh a$ and from lemma 5.3 above we have $\cosh z>$ $\operatorname{coth} a$, we conclude that

$$
d S / d x<\operatorname{coth} z+\operatorname{coth} x
$$

Now we turn to the second pentagon to get a third inequality. By Lemmas 5.4 and 5.5, we see that $\partial S / \partial y=(1-\mu) \cos (\pi-\alpha)=-(1-\mu) \cos (\alpha)$ and $\partial S / \partial z=\sin (\beta) \cosh ((1-\lambda) a)$. Thus $d S / d x=-(1-\mu) \cos (\alpha) \frac{d y}{d x}+(\partial S / \partial x)+$ $\sin (\beta) \cosh ((1-\lambda) a) d z / d x$. Since $d z / d x<0$, the third term is negative and the first term is at most $\left|\frac{d y}{d x}\right|<\operatorname{coth} x$. Hence

$$
d S / d x<(\partial S / \partial x)+\operatorname{coth} x .
$$

To bound the first term we want to use Lemma 5.5 above. Let $P$ be the vertex between $S$ and $(1-\lambda) a$. Draw the perpendicular from $P$ to $x$ and call the foot of the perpendicular $Q$. Let $r$ be the distance from side $z$ to $Q$. Clearly $(1-\lambda)>r$ since $r$ is the shortest distance between two geodesics. Applying Lemma 5.5 to the quadrilateral with sides $P Q, x-r,(1-\mu) y$ and $S$ shows $\partial S / \partial(x-r)<\operatorname{coth}((x-r) / 2)$. But $\partial S / \partial x=\partial S / \partial(x-r)$ as we can make the infinitesimal change of $(x-r)$ at the end point other than $Q$. Hence,

$$
d S / d x<\operatorname{coth}((x-r) / 2)+\operatorname{coth} x
$$


Now we show that if there is $x$ so that $d S / d x>M+\operatorname{coth} x$ for some constant $M$, then $M<3$. Thus $d S / d x \leq 3+\operatorname{coth} x<4 \operatorname{coth} x$. By (2) we see $\operatorname{coth} z>$ $M$. From lemma 5.3, we have $\cosh x>\operatorname{coth} z$. Hence $x>\operatorname{arccosh} M$. From (4) we see $\operatorname{coth}((x-r) / 2)>M$ and hence $r>x-2 \operatorname{arccoth} M$. Hence $(1-\lambda) a>$ $r>x-2 \operatorname{arccoth} M$. From (1) we have $1>M \sinh ((1-\lambda) a) \sinh z$. By lemma 5.3 again, we have $\sinh x \sinh z>1$. Hence

$$
1>M \sinh ((1-\lambda) a) / \sinh x>M \sinh (x-2 \operatorname{arccoth} M) / \sinh x .
$$

Since $d(\log (\sinh t)) / d t=\operatorname{coth} t$ is a decreasing function of $t$, we know $\sinh (t-$ $c) / \sinh t$ is an increasing function of $t$ therefore

$$
\begin{aligned}
1 & >M \sinh (\operatorname{arccosh}(M)-2 \operatorname{arccoth} M) / \sqrt{M^{2}-1} \\
& =M\left(M^{2}+1\right) /\left(M^{2}-1\right)-2 M^{3} /\left(M^{2}-1\right)^{3 / 2} .
\end{aligned}
$$

Thus we get a contradiction if $M \geq 3$. Thus $d S / d x<4 \operatorname{coth} x$ and we are done.

\section{References}

[1] A Beardon, The geometry of discrete groups, Springer-Verlag, Berlin-New York (1983)

[2] F Bonahon, Bouts des variétés hyperboliques de dimension 3, Ann. of Math. 124 (1986) 71-158

[3] F Bonahon, Earthquakes on Riemann surfaces and on measured geodesic laminations, Trans. Amer. Math. Soc. 330 (1992) 69-95

[4] $\mathbf{P}$ Buser, Geometry and spectra of compact Riemann surfaces, Birkhäuser, Boston (1992)

[5] M Dehn, Papers on group theory and topology, J. Stillwell (editor), SpringerVerlag, Berlin-New York (1987)

[6] A Fathi, F Laudenbach, V Poenaru, Travaux de Thurston sur les surfaces, Astérisque 66-67, Société Mathématique de France (1979)

[7] J Hubbard, H Masur, Quadratic differentials and foliations, Acta Math. 142 (1979) 221-274

[8] Y Imayoshi, Y. and M Taniguchi, An introduction to Teichmüller spaces, Translated and revised from the Japanese by the authors, Springer-Verlag, Tokyo (1992)

[9] F Luo, Simple loops on surfaces and their intersection numbers, preprint (1997)

[10] F Luo, R Stong, Dehn-Thurston coordinates of curves on surfaces, preprint (2002) 
[11] A Papadopoulos, On Thurston's boundary of Teichmüller space and the extension of earthquakes Topology Appl. 41 (1991) 147-177

[12] R Penner, J Harer, Combinatorics of train tracks, Annals of Mathematics Studies, 125, Princeton University Press, Princeton, NJ (1992)

[13] W Thurston, On the geometry and dynamics of diffeomorphisms of surfaces, Bull. Amer. Math. Soc. 19 (1988) 417-438

[14] W Thurston, Geometry and topology of 3-manifolds, Princeton University lecture notes (1976) 